J. Clin. Chem. Clin. Biochem.

Vol. 17, 1979, pp. 241-246

\title{
Anwendung eines Computerprogramms zur Berechnung radioimmunologischer Meßwerte
}

\author{
Von W. Huber, K. Mann und H.-J. Karl
}

Aus der Medizinischen Klinik II des Klinikums Großhadern, München

(Eingegangen am 12. Juni/13. November 1978)

Zusammenfassung: Basierend auf dem Computerprogramm von Marschner et al. ((1974), Årztl. Lab. 20, 184-191) zur Auswertung von Radioimmunoassay-Kurven mittels Spline-Funktionen wurde das Programm hinsichtlich der sofortigen Verfügbarkeit wichtiger Qualitätsparameter des Assays verbessert und an den Rechner Siemens 4004/151 angepaßt. Eine Erweiterung des Fortranprogramms ermöglicht jetzt eine genau reproduzierbare und von objektiven Kriterien abhängige Glättung der Spline-Funktionen. Der weitgehend selbständige Programmablauf macht EDV-ausgebildetes Personal zur rechengesteuerten Radioimmunoassay-Auswertung entbehrlich.

\section{Computerized data processing for radioimmunoassay}

Summary: The computer programm of Marschner et al. ((1974), Ärtl. Lab. 20, 184-191) for the calculation of radioimmunoassay data by spline functions was improved with regard to immediate availability of quality parameters. The existing programm was adapted to a computer Siemens 4004/151. Methods are reported for obtaining an exactly reproducible and objective smoothing of spline functions. The additional modifications enable even unskilled technicians to use the programm.

\section{Einleitung}

Die methodische Überlegenheit der „Spline-Approximation" gegenüber allen Linearisierungs- und Regressionsverfahren bei der radioimmunologischen Meßwertberechnung wurde in der Literatur $(1,2,3)$ ausführlich dargelegt.

Hauptargument zu Gunsten der Spline-Funktionen ist das Fehlen spezieller Voraussetzungen über den Kurvenverlauf. Ḋarüber hinaus liefert dàs Verfahren Parameter, die zu einer objektiven Beurteilung der Qualität der Kurve herangezogen werden können.

Ausgehend von der großen Probenżahl und der mathematisch anspruchsvollen Spline-Approximation wurde von Marschner et al. (1) ein Rechenprogramm in Fortran IV erstellt, das die Grundlage dieser Arbeit war.

Ziel der Modifikation und Weiterentwicklung am Programm waren fólgende Punkte:

1. Umstellung auf den Rechenbetrieb an einem Rechner Siemens 4004/151 mit dazugehöriger Peripherie wie Lochstreifenleser, Terminal, Plattendateien und Schnelldiucker.

2. Automatisierung des Programmablaufs, so daß während des Rechenvorgangs vom Benutzer keine Daten oder Befehle eingegeben zu werden brauchen.
3. Reproduzierbare und objektiven Kriterien gehorchende Glättung der Spline-Funktionen unter Berücksichtigung der Erfordernisse der quantitativen Auswertung.

4. Ausgabe verschiedener Parameter zur Beurteilung der Qualität der Meßwerte und zur langfristigen Dokumentation der Assay-Stabilität.

\section{Datenhandhabung}

Abbildung 1 zeigt schematisch den Ablauf der radioimmunologischen Meßwertbestimmung.

Lochstreifendaten werden auf eine Plattendatei übertragen. Korrekturen werden mit einem Datei-Bearbeitungsprogramm am Terminal vorgenommen. Während des Ablaufs des Computerprogramms ist kein Eingriff in den Rechenbetrieb nötig.

Zur Kontrolle des Ablaufs werden am Bildschirm wichtige Kennwerte und formale Fehlermeldungen gezeigt, so daß das Programm gegebenenfalls mit geänderten Daten neu gestartet werden kann.

Im Anschluß an die Berechnung erfolgt die Ausgabe der Ergebnisse auf dem Schnelldrucker (numerische Werte und graphische Darstellungen). Für die routinemäßige Auswertung von etwa 500 Proben werden ein- 


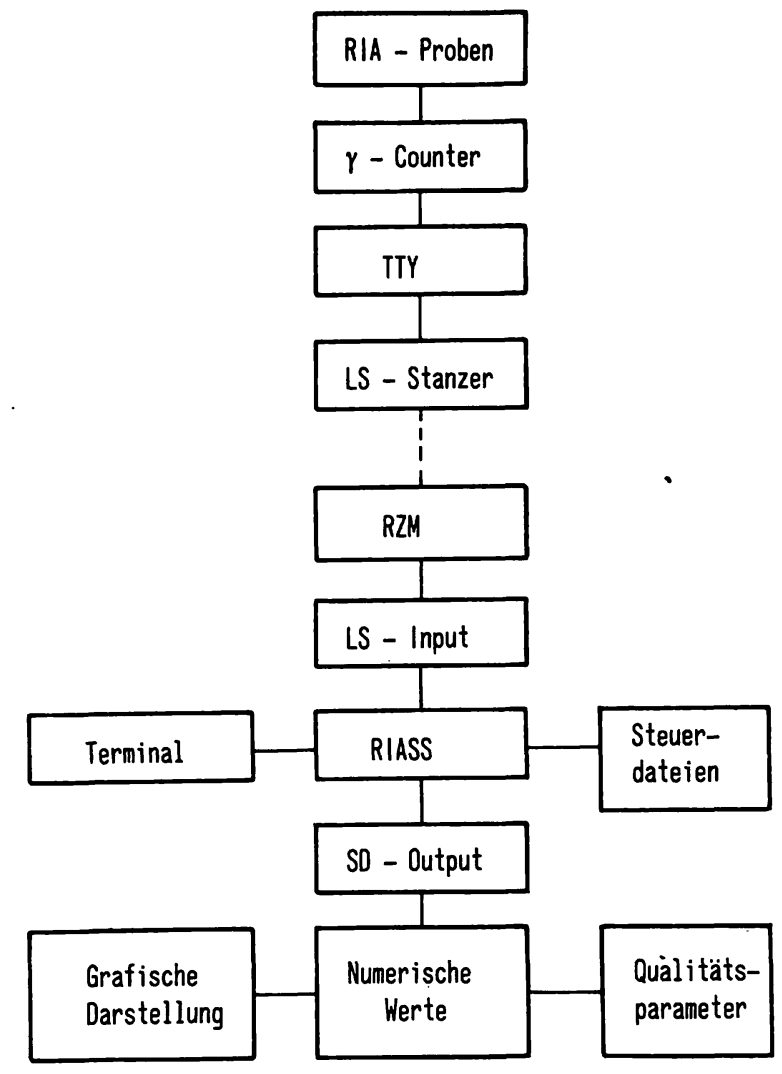

Abb. 1. Schema der RIA-Auswertung mit Computer.

\begin{tabular}{|c|c|}
\hline \multicolumn{2}{|c|}{ Verwendete Abkürzungen: } \\
\hline$\gamma$-Counter & $\begin{array}{l}\text { : Automatisches Zählgerät für radiomar- } \\
\text { kierte Proben }\end{array}$ \\
\hline LS & : Lochstreifen \\
\hline & $\begin{array}{l}\text { : Fernschreiber mit LS-Stanzer } \\
\text { : Lesegerät für LS }\end{array}$ \\
\hline RZM & $\begin{array}{l}\text { : Rechenzentrum des medizinischen Fach- } \\
\text { : bereichs }\end{array}$ \\
\hline RIASS & $\begin{array}{l}\text { : Rechenprogramm zur Auswertung der } \\
\text { Zählergebnisse }\end{array}$ \\
\hline & : Schnelldrucker zur Ergebnisausgabe \\
\hline
\end{tabular}

schließlich Datei-Korrekturen etwa 15-20 Minuten benötigt. Die reine Rechenzeit beträgt etwa 12 Sekunden und die Ergebnisse des Radioimmunoassays sind innerhalb 45 Minuten an der Schnelldruckerausgabe im Rechenzentrum verfügbar.

\section{Qualitätsparameter und Kennwerte}

Wichtiger Bestandteil des Programms ist es, Auskunft über die Präzision und die Sensitivität des jeweiligen Assays zu geben. Ein besonderer Vorteil der SplineApproximation besteht darin, daß zur Gewinnung von globalen Qualitätsparametern nur geringer Rechenaufwand erforderlich ist.

Zur raschen und sicheren Beurteilung des Assays hat es sich bewährt, 10 wesentliche Kennzahlen heranzuziehen. Im Folgenden sei deren Bedeutung für die Qualitätskontrolle erläutert.

Auf dem Schnelldrucker ausgegeben werden die spezifische und unspezifische Bindung, der arithmetische
Mittelwert der Zählergebnisse von Gesamtaktivitäten, Standardleerwerten und Standardantigen-Konzentrationen jeweils mit Standardabweichung und Variationskoeffizient.

Die untere Nachweisgrenze des Assays wird definitionsgemäß als dreifache Standardabweichung des gemittelten Standardleerwerts in Prozent und in Antigenkonzentration angegeben. Diejenige Antigenkkonzentration, bei der gerade die Hälfte des radiomarkierten Antigens gegenüber dem Standardleerwert verdrängt worden ist, wird als 50 Prozent-Intercept ausgedruckt (Abb. 2).

Bestimmung des Glättungsfaktors durch sukzessive Spline-Approximation

Charakteristisches Merkmal der Spline-Approximation ist die Wichtung der Meßwerte sowie die Angabe eines zunächst frei wählbaren Glättungsfaktors. Als Maß für die „Gültigkeit" eines Meßwertes wird hier der Variationskoeffizient einer Mehrfachbestimmung herangezogen. Je kleiner dieser Koeffizient ausfällt, um so mehr wird dieser Meßpunkt beim Verlauf der Standardkurve berücksichtigt.

Die Wahl eines „optimalen“ Glättungsfaktors ist im engeren Sinne kein mathematisches Problem, sondern der Kompromiß zwischen konkurrierenden Forderungen. Einerseits soll die Kurve die Meßreihe wirklich darstellen, d. h. die Rückinterpolierbarkeit der Meßpunkte muß gewährleistet sein. Andererseits soll die Kurve keine Oszillation oder abrupte Richtungswechsel aufweisen. Es ist ineffizient, wenn der optimale Glättungsfaktor nur von der Anzahl der Meßpunkte abhängig gemacht und der geeignete Glättungsfaktor lediglich durch Austesten bestimmt wird (1).

\section{Kriterien zur Optimierung des Glättungsfaktors}

Im allgemeinen gehen in die Wahl des Glättungsfaktors die Zahl der Meßpunkte, deren Wichtung, die Präżision des Assays sowie der formale Kurvenverlauf ein. Über die beiden letzten Kriterien läßt sich a priori keine Aussage machen, da sie ja erst nach der rechnerischen Auswertung zur Verfügung stehen. Eine feste Wahl des Glättungsfaktors ist daher stets problematisch und wird in vielen Fällen dem Problem der Kurvenglättung nicht gerecht.

Die automatische Bestimmung eines optimalen Glättungsfaktors läuft im Programm so ab, daß zunächst geprüft wird, ob ein sigmoidaler Verlauf der Kurve zu erwarten ist. Dazu wird abgefragt, ob es mindestens einen Zählratenmittelwert einèr Standardprobe gibt, der größer als der des Standardleerwertes ist. Wenn ja, darf die Kurve maximal einen relativen Extremwert besitzen, wenn nein, dàrf sie keinen besitzen. Solange diese alternative Forderung nicht erfullt ist, wird der Glättungsfaktor beginnend mit einem festen Startwert von 0,2 schrittweise um einen konstanten Faktor erhöht, bis die Kurve soweit geglättẹt ist, dạß keine wei- 


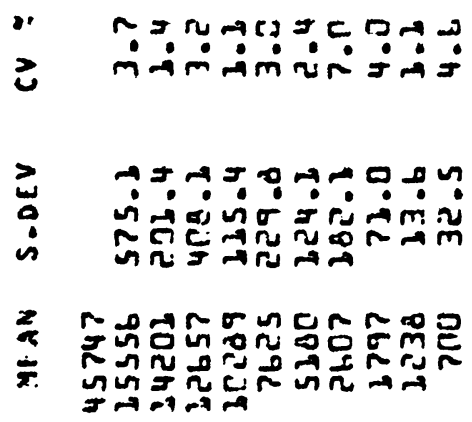

míceconde=0 กี่

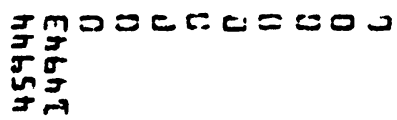

moco0000000 $\underset{\sim}{\sim} \underset{\sim}{\sim}$ जु

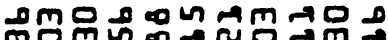

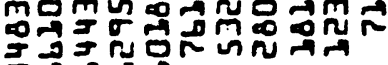

走

นก

Oก⿻尸丨

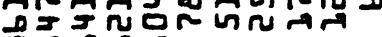

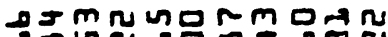

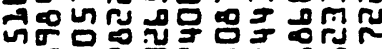
जin

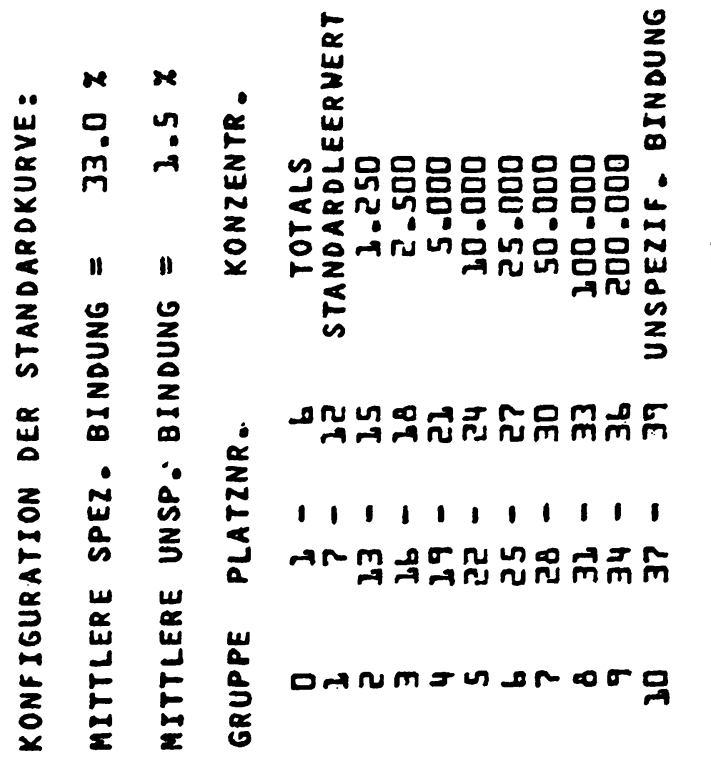

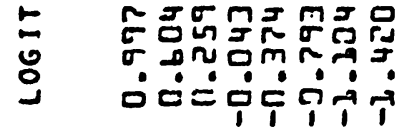

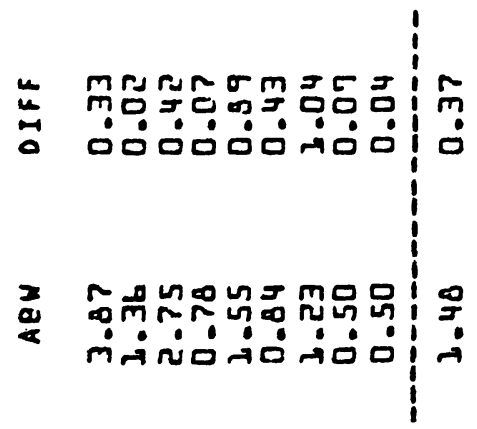

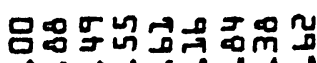

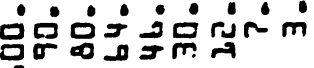

索
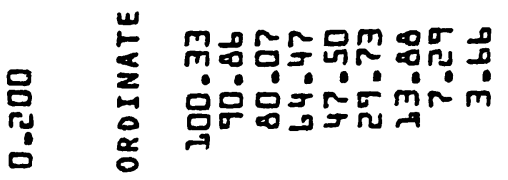

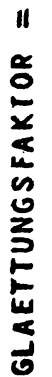

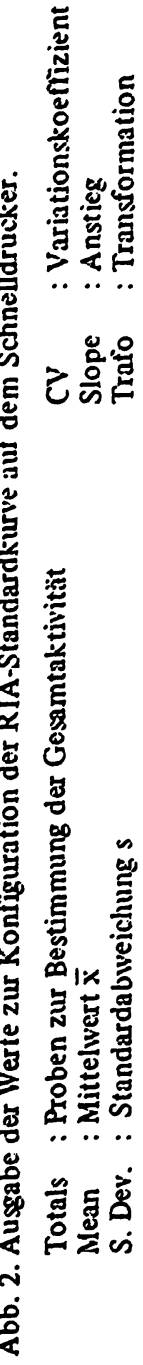




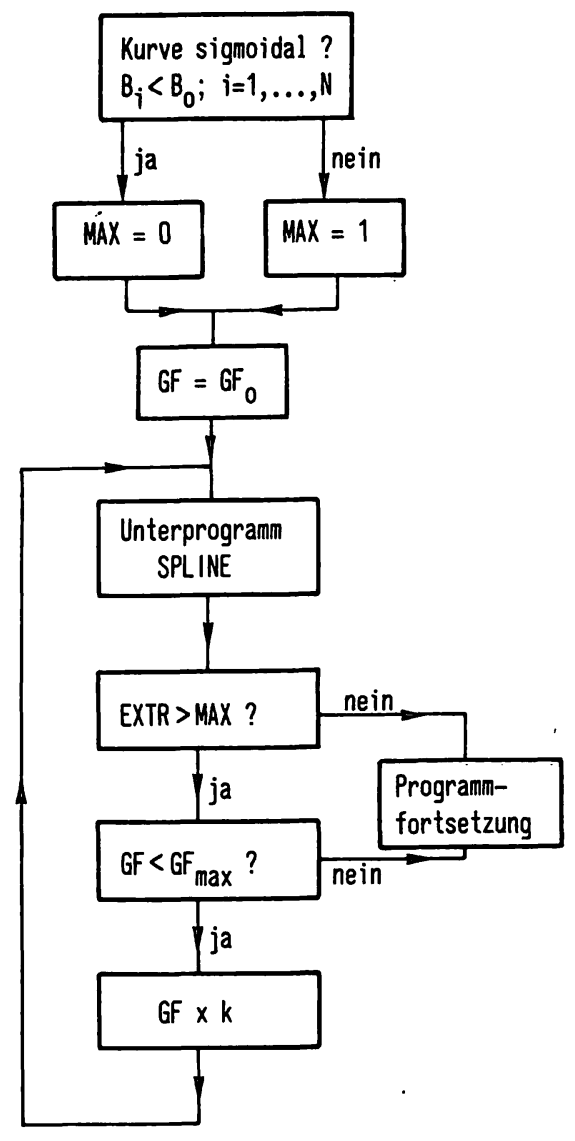

Abb. 3. Algorithmus zur programmgebundenen Bestimmung des Glättungsfaktors.

Verwendete Abkürzungen:

$B_{0} \quad$ : Zählrate des Standardleerwertes

$\mathrm{B}_{\mathbf{i}} \quad:$ Zählrate der Standardproben

$\mathrm{N}$ : Zahl der Meßpunkte

MAX : Maximale Anzahl der Extremwerte

GF : Glättungsfaktor

EXTR : Zahl der tatsächlichen Extremwerte

$\mathrm{GF}_{\mathrm{O}}$ : Startwert für GF $(=0,2)$

$\mathrm{GF}_{\max }$ : Obere Grenze für GF $(=5,0)$

k : Konstanter Faktor $(=1,5)$

teren Extremwerte auftreten. Die Erhöhung des Glättungsfaktors geschieht solange, bis eine sinnvolle Obergrenze $(5,0)$ erreicht wird. Jenseits dieser Grenze entfernt sich die Kurve soweit von den Meßwerten, daß ein Ablesen an solchen Standardkurven nicht mehr gerechtfertigt ist. Sollte die Extremwert-Forderung bis dahin nicht erfültt worden sein, wird mit dem zuletzt errechneten Glättungsfaktor weitergearbeitet (Abb. 3).

Mathematischer Aspekt der Prüfung auf Extremwerte

Im Intervall $i$ ist die Standardkurve gegeben durch das kubische Polynom

$$
\mathrm{Y}=\mathrm{A}_{\mathrm{i}}+\dot{B}_{\mathrm{i}} \mathrm{X}+\mathrm{C}_{\mathrm{i}} \mathrm{X}^{2}+\mathrm{D}_{\mathrm{i}} \mathrm{X}^{3}, \quad \mathrm{X}_{\mathrm{a}} \leqslant \mathrm{X}<\hat{\mathrm{X}}_{\mathrm{e}}
$$

wobei

Y Ordinatenwerte

$\mathrm{X}$ Abszissenwerte

$A_{i}, B_{i}, C_{i}, D_{i}$ Spline-Koeffizienten

$\mathrm{X}_{\mathrm{a}}$ Intervallanfang

$\mathrm{X}_{\mathrm{b}}$ Intervallende

Besitzt nun die erste Ableitung

$$
\mathrm{Y}^{\prime}=\mathrm{B}_{\mathrm{i}}+2 \mathrm{C}_{\mathrm{i}} \mathrm{X}+3 \mathrm{D}_{\mathrm{i}} \mathrm{X}^{2}
$$

eine Nullstelle mit einer Lösung im Intervall i, so daß gilt

$$
\mathrm{Y}^{\prime}=0 \text { mit } \mathrm{X}_{\mathrm{a}} \leqslant \mathrm{X}_{1,2}<\mathrm{X}_{\mathrm{e}}
$$

so ist (mindestens) ein Extremwert (und evtl. ein Sattelpunkt) aufgefunden.

Die Lösung lautet explizit:

$$
X_{1,2}=\left(-C_{i} \pm \sqrt{C_{i}^{2}-3 B_{i} C_{i}}\right) / 3 D_{i} .
$$

Der zusätzliche Rechenaufwand zur Extremwertbestimmung konnte damit sehr gering gehalten werden.

\section{Glättungsfaktor als Qualitä tsparameter}

Wird der Glättungsfaktor auf programmgebundene Weise bestimmt, so ist er nicht länger ein willküllich wählbarer Parameter. Als exakt reproduzierbarer Kennwert, in den Wichtung, Meßgenauigkeit und formaler Verlauf der Kurve eingehen, wird der Glättungsfaktor nun ein wichtiger Qualitätsparameter mit globalem Charakter. Eine mit diesem Verfahren erstellte und auf Schnelldrucker ausgegebene Standardkurve zeigt Abbildung 4.

\section{Weitere Qualitätsparameter}

Der mit einer Mehrfachbestimmung ermittelte Meßpunkt ist um so ,glaubwürdiger", je geringer seine Standardabweichung ist. Ein Maß für die Qualität der gesamten Kurve ist deshalb eine auf den Standardleerwert und die Zahl der Meßpunkte normierte Summe der Abweichungsquadrate. Brauchbare numerische Werte dieser Summe liegen zwischen 1-3 (Vergleiche Abb. 2 mit der Abweichung = 1,48).

Bedingt durch die Spline Approximation stimmt der Mittelwert der Zählrate nicht exakt mit dem dąugehörigen Ordinatenwert der Interpolationskurve überein. Die Summe der Absolutbeträge dieser Differenz, noimiert auf die Zahl der Meßpunkte, stellt somit ein Kriterium für die Rückinterpolierbarkeit der Standardkurve dar. Numerische Erfahrungswerte liegen zwischen 0,3-1 (Vergleiche Abb. 2 mit Differenz = 0,37).

\section{Logit-Transformation}

Beschreibt man die Dosisvariable in linearer Abhängigkeit des Åntigens, so genügen 2 Parameter, nämlich Achsenabschnitt (Intercept) und Steigung. (Slope), um die gesamte Kurve (Gerade) charakterisieren zu können. 


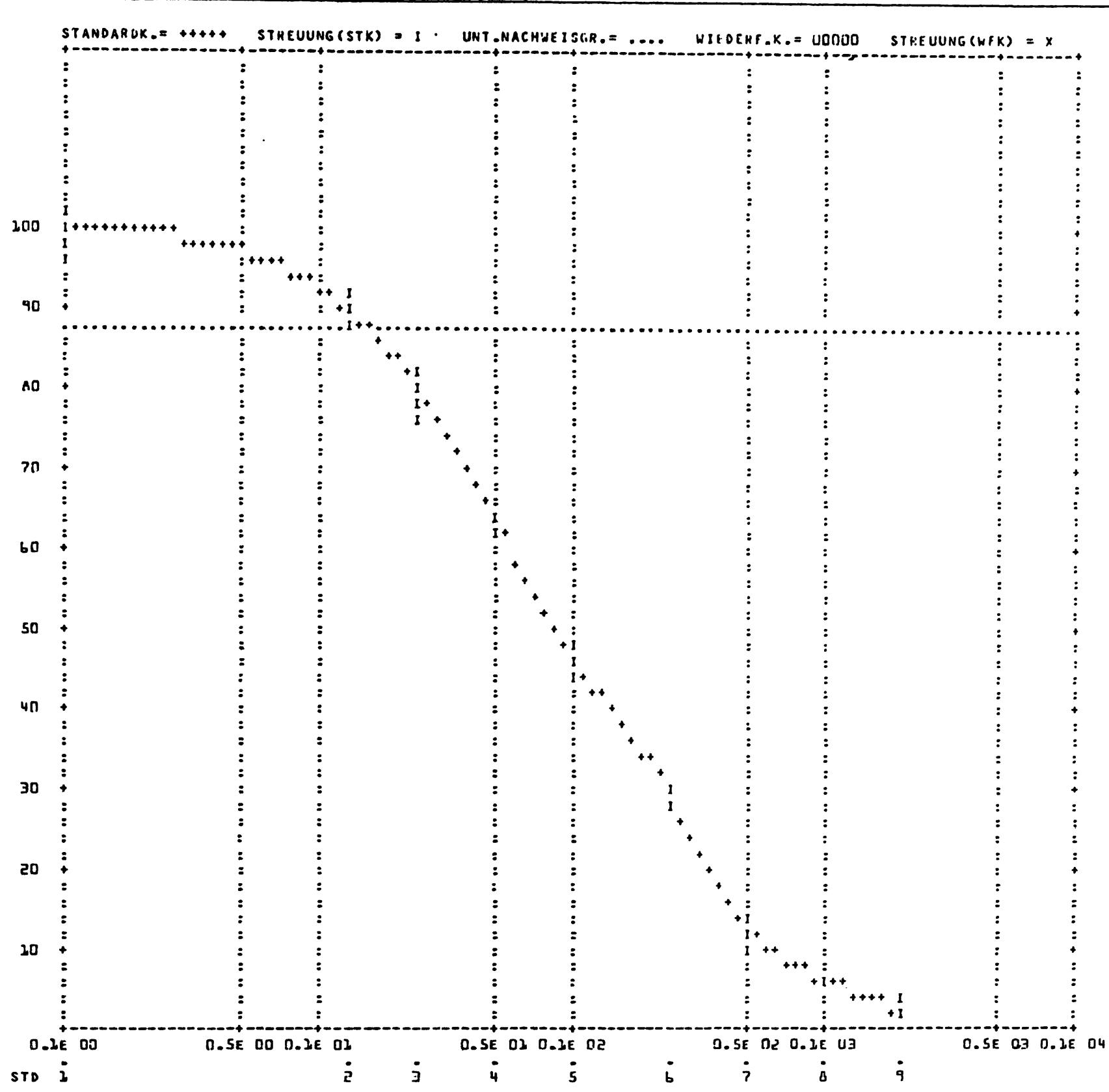

Abb. 4. Schnelldruckerausgabe der Standardkurve

Abszisse: Antigenkonzentration in logarithmischer Teilung und normierter Schreibweise mit Mantisse und Exponent. $0.5 \mathrm{E} 01$ bedeutet z.B. $5 \mu \mathrm{g} / \mathrm{l}$. Die einzelnen Meßpunkte sind markiert und fortlaufend numeriert. Dem Standardleerwert (Meßpunkt Nr. 1) wird programmgebunden eine geeignete Konzentration zugeordnet (hier $0,1 \mu \mathrm{g} / \mathrm{l}$ ), da wegen der Logarithmierung die Konzentration nicht Null sein darf.

Ordinate: Prozentualer Anteil gebundenen, radioaktiv markierten Antigens, bezogen auf den Standardleerwert nach der Formel:

$$
\begin{aligned}
& (\mathrm{B}-\mathrm{N}) /\left(\mathrm{B}_{\mathrm{O}}-\mathrm{N}\right) \times 100 \% \\
& \mathrm{~B}: \text { Zählrate einer Standardprobe } \\
& \mathrm{B}_{\mathrm{O}}: \text { Zählrate des Standardleerwertes } \\
& \mathrm{N}: \text { Zählrate der unspezifischen Bindung } \\
& \text { STD : Standard }
\end{aligned}
$$

Um auf diesen Vorteil trotz Spline Approximation nicht verzichten zu müssen, werden im Programm die Ordinatenwerte $Y$, d. h. die nach der Interpolation gewonnenen Werte der Standardkurve, einer Logit-Transformation unterzogen:

$$
\log (Y /(100 \%-Y))
$$

mit der Nebenbedingung $1 \% \leqq \mathrm{Y} \leqq 99 \%$
Anschließend wird eine lineare Regression zwischen diesen Logit-Werten und den Logarithmen der Abszissenwerte (Konzentration des unmarkierten Antigens) durchgeführt. Die sich daraus ergebende Steigung der Ausgleichsgeraden (Slope) sowie der dazugehörige Korrelationskoeffizient werden ausgegeben (Abb. 2). Der Slope der Regressionsgeraden gestattet einen unmittelbaren Test der Assay-Kurven auf Parallelität. Der 
Korrelationskoeffizient gibt Auskunft über die Sigmoidalität der Standardkurve, d. h. wie gut sich die LogitTransformation durchführen läßt. Eine ideal sigmoide Kurve erzielt einen Korrelationskoeffizienten von -1 .

\section{Qualitätskontrolle}

Eine rasche und übersichtliche Information über die zeitliche Stabilität einer Assay-Reihe gibt die Kontrollkarte $(4,5)$. Die Abszisse bilden hier die Assays in ihrer chronologischen Reihenfolge, während auf der Ordinate verschiedene Qualitätsparameter und Meßergebnisse eingetragen werden (Prolactin-Kontrollkarte Abb. 5).

Deutlich zu erkennen ist hierbei, daß die Assays mit den Nummern 6, 7 und 8 ,Ausreißer" sind, was sich in diesem Fall auf ein Antigen anderer Herkunft im RIA-Kit des Herstellers zurückführen ließ.

\section{Diskussion}

Mit dem erweiterten Rechenprogramm steht eine Auswertungsmethode für radioimmunologische Meßwerte zur Verfügung, die der Auswertung durch freie Wahl des Glättungsfaktors an Schnelligkeit und Reproduzierbarkeit überlegen ist. Das Programm liefert in Form spezieller Parameter Entscheidungshilfen zur sicheren Beurteilung der Assayqualität sowie zur langfristigen Dokumentation der Stabilität. Im Falle käuflicher RIA-Kits können Unregelmäßigkeiten der gelieferten Substanzen zweifelsfrei nachgewiesen werden. Bei der Entwicklung eigener Assays stellen die Parameter eine wertvolle Hilfe zur Optimierung der Assays dar.

Unter Verwendung der Spline-Approximation mit einem objektiven Glättungsalgorithmus sowie der raschen Verfugbarkeit von Meßwerten und Qualitätsparametern wurde eine universelle und nahezu voraussetzungslose Methode zur rechnergesteuerten Interpretation von RIA-Kurven entwickelt. Der weitgehend selbständige Programmablauf macht speziell ausgebildetes Personal entbehrlich. Vielmehr können auch technische Hilfs-

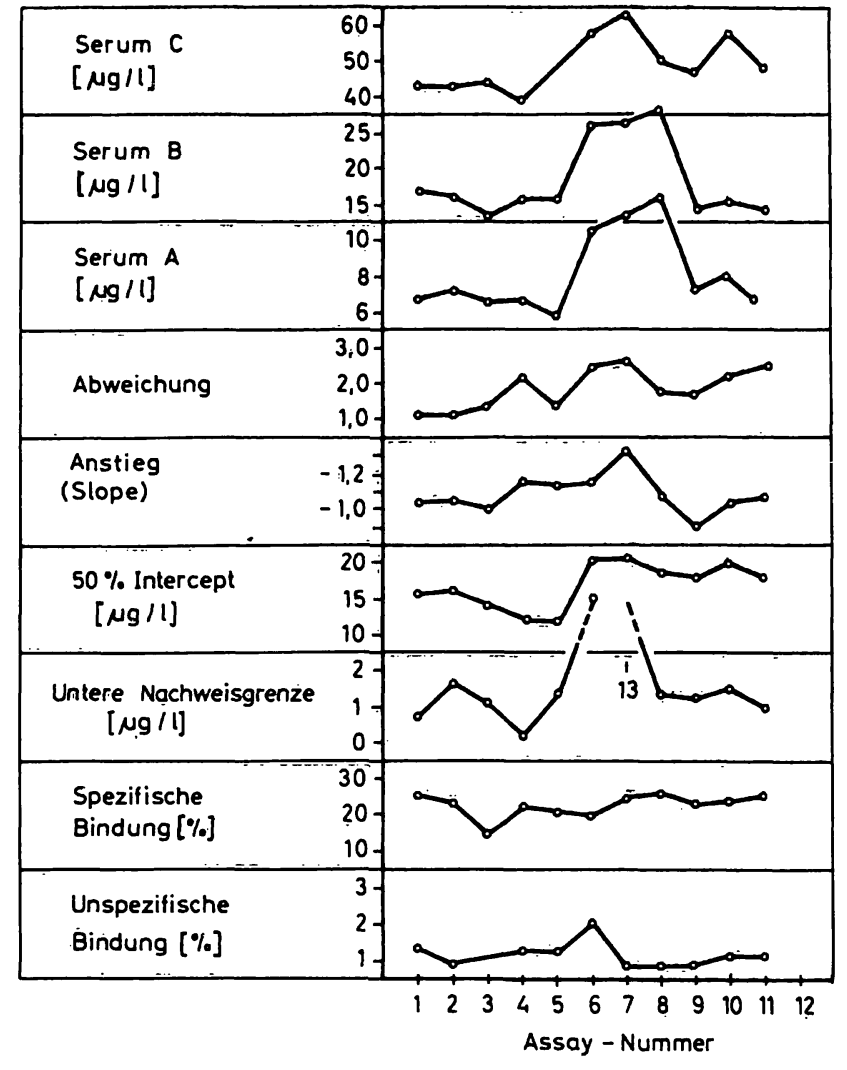

Abb. 5. Kontrollkarte für Prolactin-Assays

Abszisse: Assays in chronologischer Reihenfolge Ordinate: Kennwerte, Qualitätsparameter und Kontrollbestimmungen

kräfte in die Rechner-Auswertung ohne Schwierigkeiten eingewiesen werden.

\section{Danksagung}

Für die freundliche Überlassung des Rechnerprogramms möchten wir uns bei Prof. Dr. P. C. Scriba, Dr. med. Marschiner und Dr. Bauer der Med. Klinik Innenstadt der Universität München bedanken. Weiterhin gilt unser Dank Herrn Dr. Schrag vom Rechenzentrum des medizinischen Fachbereichs der Universität in Großhadern, der uns bei Problemen der Datenverarbeitung hilfreich beriet.

\section{Literatur}

1. Marschner, I., Dobry, I. H., Erhardt, F., Landesdorfer, T., Popp, B., Ringel, C. \& Scriba, P. C. (1974), Ärztl. Lab. 20 , 184-191.

2. Nolte, H., v. z. Mühlen, A. \& Hesch, R. D. (1976), diese Z. $14,253-259$.

3. Reinsch, Ch. H. (1967), Numer. Math. 10, 177-183.

4. Rodbard, D., (1974), Clin. Chem. 20, 1255-1270.

5. Rodbard, D., Rayford, P. L., Cooper, J. A. \& Ross, G. T. (1968), J. Clin. Endocrinol. Metab. 28, 1412-1418.

\author{
Dr. K. Mann \\ Med. Klinik II \\ Klinikum Großhadern \\ Marchioninistraße 15 \\ D-8000 München 70
}

